\title{
Socioeconomic restraints and brain tumor surgery in low-income countries
}

\author{
Ahmed E. Helal, MBBCh, Heba Abouzahra, MBBCh, Ahmed Abdelaziz Fayed, MD, \\ Tarek Rayan, MD, and Mahmoud Abbassy, MD
}

Department of Neurosurgery, Alexandria University, Alexandria, Egypt

\begin{abstract}
Healthcare spending has become a grave concern to national budgets worldwide, and to a greater extent in low-income countries. Brain tumors are a serious disease that affects a significant percentage of the population, and thus proper allocation of healthcare provisions for these patients to achieve acceptable outcomes is a must.

The authors reviewed patients undergoing craniotomy for tumor resection at their institution for the preceding 3 months. All the methods used for preoperative planning, intraoperative management, and postoperative care of these patients were documented. Compromises to limit spending were made at each stage to limit expenditure, including low-resolution MRI, sparse use of intraoperative monitoring and image guidance, and lack of dedicated postoperative neurocritical ICU.

This study included a cohort of 193 patients. The average cost from diagnosis to discharge was $\$ 1795$ per patient (costs are expressed in USD). On average, there was a mortality rate of $10.5 \%$ and a neurological morbidity rate of $14 \%$, of whom only $82.2 \%$ improved on discharge or at follow-up. The average length of stay at the hospital for these patients was 9.09 days, with a surgical site infection rate of only $3.5 \%$.
\end{abstract}

The authors believe that despite the great number of financial limitations facing neurosurgical practice in low-income countries, surgery can still be performed with reasonable outcomes.

https://thejns.org/doi/abs/10.3171/2018.7.FOCUS18258

KEYWORDS socioeconomic restraints; brain tumor complications; low-income countries

$\mathrm{H}$ EALTHCARE budgets have grown to represent a large burden on national expenditure worldwide. In the United States, the healthcare expenditure for 2016 reached $\$ 3.3$ trillion or $\$ 10,348$ per person, representing $17.9 \%$ of gross domestic product. ${ }^{3}$ With the expanding technological advances in medicine, especially in the Western world, it has been important to scrutinize the cost-effectiveness of the use of new advances in dayto-day medical practice. On the contrary, low-income countries, when made aware of the luxury of healthcare in high-income countries, have been forced to attempt to provide similar clinical outcomes regarding mortality and morbidity, on a limited budget.

Brain tumors affect 28.57 per 100,000 individuals each year in the United States. ${ }^{19}$ They represent extremely critical and financially demanding patients, with highly variable outcomes and prognoses. At a tertiary care institution in a low-income country, demands of comparable outcomes while accepting the restraints enforced by very limited healthcare spending require extremely judicious use of finances to serve a large patient population.

Some tools have been shown to improve outcomes, such as cranial nerve monitoring in cerebellopontine angle tumors, which has allowed a significant improvement in preservation of facial nerve function, ${ }^{5,9,11,28}$ and awake craniotomy and cortical mapping in low-grade glioma for increasing extent of resection, with an improvement from $54.4 \%$ to $84.6 \%$ in a single-institution sample. ${ }^{8,31}$ Mean- 
while, other tools, such as intraoperative MRI, have been shown to be of controversial benefit for increasing extent of resection. ${ }^{8}, 14$

According to the World Bank, Egypt is considered a lower- to middle-income country ${ }^{32}$ Limited data are available from developing countries to be able to assess their current treatment strategies, ${ }^{16,20}$ due to the absence or inefficiency of their brain tumor registries. Accordingly, it was essential to raise the question of whether the application of recent technological advances such as intraoperative MRI, image guidance, and other techniques in routine day-today practice is beneficial in attaining good outcomes. In our study we used in-hospital mortality and neurological morbidity rates for primary outcomes, and surgical site infection and length of stay as secondary outcomes.

\section{Methods}

After the approval of the local ethics committee in Alexandria University hospitals, we reviewed the records of patients undergoing craniotomy for tumor resection at our institution over a period of 3 months. We collected data regarding average direct costs of the patient care from admission to discharge, length of stay, and patient outcomes. The primary outcome measure used was in-hospital mortality.

Diagnosis of patients with brain tumors includes an initial 16-slice CT scan in most cases and a contrast-enhanced MRI brain scan. The majority of the patients in our study had their MRI done in low-power, open MRI scanners of $0.2 \mathrm{~T}$ to $0.5 \mathrm{~T}$ in order to minimize costs. Only a small percentage of our study patients had a 1.5-T or 3.0T MRI scan, with functional imaging as required.

The cases that were excluded from surgeries in our setting included recurrent high-grade gliomas with Karnofsky Performance Scale score below 70, and patients presenting with brain metastases who had uncontrolled primary disease or multiple brain masses. The decision about the extent of resection was planned according to each case, considering the risk to the patients' lives or their morbidity, and keeping in mind the limited intraoperative equipment. For example, a small residual amount of a medial sphenoid wing meningioma is accepted if it is stuck to the internal carotid artery.

Intraoperative preparation of the patient included positioning without the use of any head fixation, but with a simple doughnut-shaped headrest and rolled-up drapes. Draping was done using reusable textile drapes and gowns. Craniotomy was done using Hudson's burr hole and Gigli's bone saw. Disposable drill bits were used more than once after resterilization.

No image guidance was used in any of our patients except for 3 individuals, for which there were enough funds to rent the equipment from an outside source for approximately $\$ 400$ (costs are expressed in USD throughout). Instead, for localization we relied on bony landmarks and surface anatomy of the brain. Intraoperative monitoring was used for approximately $4 \%$ of cases. Both image guidance and neurophysiological systems are rented by several hospitals in our region for highly selected cases; this is to overcome the expensive price and maintenance.
Intraoperative supplies such as cottonoids are hand made in our hospitals and sterilized to avoid additional costs. Each cottonoid is sewn using 2-0 silk sutures that act as a marker to avoid losing it in the tumor cavity; they are cut in different sizes and ironed before packing and sterilization. The needle of an insulin syringe was used as a disposable arachnoid knife. Sterile intravenous infusion catheters were fashioned and used as a suction regulator. Repositioning of the bone flap was done using absorbable sutures to avoid the cost of using miniplates and screws.

Postoperative care for patients was provided by a basically equipped general ICU with no dedicated neurocritical care nursing staff or equipment. No intracranial pressure, perfusion, or electroencephalography monitoring was available in the ICU.

\section{Results}

After reviewing patient records for the preceding 3 months, we found that 193 craniotomies for tumor resection were performed at the Alexandria main university hospital and affiliated hospitals. These were all patients with brain tumors who were admitted to our department through the outpatient clinic and the emergency department. The most common tumor type was meningiomas (30.5\%), followed by gliomas, both low- and high-grade types (25.9\%). The third most common tumor type was pituitary adenomas $(9.8 \%)$, followed by metastatic disease (representing $7.3 \%$ of our study cases). The mean age of patients who underwent operation at our department was 42 years, with a maximum age of 76 years and a minimum of 2 years. The average cost from diagnosis to discharge was $\$ 1795$ per patient. The total cost per patient showed a wide variation, ranging from as low as $\$ 900$ per patient to as much as $\$ 2400$. This sum includes imaging costs, hospital ward stay, operating time, general ICU stay, postoperative medications, neuropathology studies, and clinic follow-up. A breakdown of standard expenses is shown in Table 1.

Over the time span of our study the in-hospital mortality rate at our institution was $10.5 \%$. Postoperative pa-

TABLE 1. Itemized cost of surgery per patient at our institution

\begin{tabular}{ll}
\hline \multicolumn{1}{c}{ Procedure } & Cost (USD) \\
\hline CT & $\$ 25$ \\
\hline MRI & $\$ 70-200$ \\
\hline Op time & $\$ 50 / \mathrm{hr}$ \\
\hline ICU stay & $\$ 200 /$ day \\
\hline Ward stay & $\$ 50 /$ day \\
\hline Medications & $\$ 300$ \\
\hline Routine laboratory tests & $\$ 50$ \\
\hline Neuropathology & $\$ 25$ \\
\hline Clinic follow-up & $\$ 70$ \\
\hline Image guidance per patient & $\$ 400$ \\
\hline Intraop monitoring per patient & $\$ 300$ \\
\hline
\end{tabular}

* This amount is an average of the medications administered during the patient's hospital stay and after discharge, including antiepileptics, antibiotics, and analgesics. 
tient neurological morbidity, including focal neurological deficits and disturbed level of consciousness, was $14 \%$. Of these patients, $42.9 \%$ improved on discharge and subsequent follow-up, whereas $39.3 \%$ were discharged with moderate disability that improved later on, and only $17.8 \%$ were left with a severe incapacitating disability. The mean length of hospital stay at our institution was on average 9.09 days. A noteworthy secondary outcome in our patient population was surgical site infection, which accounted for only $3.5 \%$ of patients.

All patients were followed at our neurosurgical clinic for the initial 2 weeks of follow-up. Further follow-up was performed at the neuro-oncology clinic, with the surgical team notified on an as-needed basis. We were able to obtain an immediate follow-up MRI sequence in only 2 cases due to financial restrictions. At the 3-month postoperative follow-up, MRI was reserved for patients with high-grade lesions. The average delay between surgery and the start of radiotherapy for patients with high-grade glioma was 8 weeks, with some patients being delayed as much as 14 weeks after surgical intervention.

\section{Discussion}

Healthcare costs and cost-effectiveness have become a global health concern, and in-depth studies of proper cost accrual are being conducted worldwide to exclude unnecessary spending. ${ }^{7,27}$ In neuro-oncology patients these matters are further highlighted due to the rapid advancement in the field of neurosurgery and the paucity of level I evidence to prove the benefit of added costs of advanced equipment in improving outcome. ${ }^{6,33}$ However, and due to the critical nature of these patients' condition, it is with great doubt that a surgeon might opt to withdraw potentially beneficial tools from his or her armament. These matters are further highlighted when it comes to neurosurgical practice in low-income countries.

A review of recent literature regarding the cost of craniotomy for tumor resection revealed that the average cost of surgery ranged from $\$ 16,977$ to $\$ 38,662$, which may increase even more in the presence of hospital-acquired complications. ${ }^{12,13,15,34,35}$ The patients toward the upper margin of cost were those requiring awake craniotomies and intraoperative monitoring. ${ }^{15}$ In comparison to our collected data, mortality rates in the literature varied from $2.4 \%$ to $4.9 \%$ at comparable Western institutions. ${ }^{1,13,23,34}$ The average length of stay in the literature ranged from a mean of 4.0 days in some studies to up to 12.1 days in other studies.1,13,25,34 Surprisingly, surgical site infection rates in the studies we reviewed reached $8 \%$ in some institutions..$^{17,19,26}$

The data presented in this study show a wide gap in per-patient spending between our institution and Western tertiary centers, and thus it would be naïve to undervalue the utility of all the tools that incur added costs. From the initial management of brain tumors, differences in patient care appear-in our institution seldom is a full MRI study with all common protocols, including diffusion-weighted, FLAIR, and susceptibility-weighted imaging, provided for all patients, but rather a limited study with few protocols is performed to provide a diagnosis and as an aid for surgi- cal planning. Furthermore, functional MRI and diffusion tensor imaging were not performed for patients with either low-grade or high-grade glioma due to the high cost of these investigations, which may have greatly affected outcomes. ${ }^{8}$

Another matter that requires consideration is the variation in the use of reusable and disposable drapes. It has become standard practice to use disposable gowns and draping in the West, whereas we still rely on reusable textile drapes at our institution. While this provides a significant reduction in costs, limited studies have failed to show significant benefits for disposable drapes over textile fabrics, and a Cochrane review has shown that in some instances plastic adhesive drapes have caused an increased risk of surgical site infection. ${ }^{18,20,31}$ These data are consistent with our results regarding surgical site infection. Another matter of cost reduction regarding operating room preparation is minimal use of 3-pin skull clamps, instead using doughnut-shaped headrests and pillows, and the lack of a dedicated surgical chair and armrests. Needless to say, craniotomy at our institution is still being performed using Hudson's burr hole and Gigli's bone saw, and repositioning of bone flaps is done using absorbable sutures. Other maneuvers, which are applied in an attempt to reduce costs without greatly affecting outcomes, include the reuse of diamond bits for drilling and at times even the use of a 1-mm Kerrison rongeur in place of the drill for anterior clinoidectomies and even anterior petrosectomies.

It has become relatively standard practice in Western institutions to use frameless stereotaxy or other forms of image guidance in brain tumor surgery, and studies have shown the increased gross-total resection outcomes and decreased morbidity facilitated by image guidance. .,32 $^{8}$ However, in our study image guidance was used in only 2 cases of glioma situated near eloquent cortex, and the equipment was rented from an outside facility that supplies several hospitals instead of being owned. A number of studies over the past decade have also highlighted the value of high-power 1.5-T or 3-T MRI in the improvement of extent of resection while minimizing postoperative morbidity in patients with intraaxial tumors; although low-power intraoperative MRI may be more economical, studies did not show significant value for its use in intraaxial tumor resection. ${ }^{8,14,32}$ Unfortunately, we were unable to use intraoperative MRI in any of our patients due to its very high cost and setup requirements.

Another intraoperative tool that has greatly enhanced outcomes of surgery for intraaxial brain tumors near eloquent cortex is awake craniotomy with intraoperative cortical mapping. This tool was seldom used in our cases, again due to its high cost relative to patient income. Similarly, neurophysiological monitoring used in many lesions involving cranial nerves or brainstem compression has been shown to decrease morbidity and is considered the standard of care. ${ }^{8,11,15,29,32}$ It is thus evident that cost reduction has a definite effect on morbidity outcomes, and on postoperative neurological deficits..$^{10,22}$

Beyond the scope of the intraoperative setting, a number of other compromises to minimize cost may have led to the difference in outcomes, including the use of generic locally made pharmaceuticals in place of the more effec- 
tive brand-name drugs. A matter that has been recently highlighted in the literature is the value of a dedicated neurocritical ICU. ${ }^{17}$ It been shown not only that a dedicated facility with staff that are aware of the needs of neurosurgical patients is of value, but also that they should be immediately available on demand. Intracranial pressure monitoring and electroencephalography were tools not readily available for our patients that have been shown to provide significant benefit.

A number of hurdles face patients at our institution for receiving postoperative adjuvant therapy. Administration of radiotherapy and chemotherapy is relatively expensive for our patients and thus most of them tend to apply for government funding to receive their oncological treatment. Although optimal timing of adjuvant therapy is a controversial matter, most studies have shown that a delay of more than 6 weeks has a significant negative effect on outcome. ${ }^{21,30}$ Accordingly, delay in adjuvant therapy caused by application processing has resulted in poor outcome in patients with high-grade lesions. Many of our patients were not able to do follow-up MRI in the immediate postoperative period as well as during the initial followup, which greatly hindered assessment of tumor residual tissue and recurrence. ${ }^{4}$ Furthermore, a number of tumor markers that have shown utility in determining response to treatment are seldom investigated due to their cost.

The availability and feasibility of the tools mentioned is not the only thing hindering outcomes at our institution; the efficiency and skill level of the personnel serving these patients is also hindered by spending limitations. Microneurosurgical skills laboratories are lacking in our institution due to the extreme costs required to set up the necessary equipment. Furthermore, proper subspecialization of neurosurgical staff is another factor, which may lead to worse outcome in comparison with what would be provided by focused, subspecialized, multidisciplinary teams. ${ }^{2,24}$ Another critical problem in our institution and moreover in our country as a whole is the proper education of nursing staff, who serve as the backbone of neurocritical patient care. Although retraining of nursing staff and technicians seems an economic step toward improving patient outcomes, we face the burden of losing welltrained staff to other countries with better opportunities and higher-paying jobs.

Although this study is fraught with many limitations in data collection and analysis, it gives us a clear notion that a significant level of spending is required to be at par with neurosurgical patient outcomes, even though not all Western institutional spending is translated into patient outcomes. Follow-up of this study should be done, including a breakdown of the tumor types and tools used and their statistical significance in changing patient outcomes. Also, a multivaried analysis should be done, including neurological and systemic morbidity as a primary outcome measure rather than mortality.

\section{Conclusions}

Healthcare expenditure greatly impacts neurosurgical practice in developing countries. Although some limitations in expenditure might affect patient outcomes, other forms of spending may not. Accordingly, the practice of craniotomy for tumor resection in low-income countries, despite being cumbersome, is still possible with a reasonable success rate and some limitations.

\section{References}

1. Barker FG II: Craniotomy for the resection of metastatic brain tumors in the U.S., 1988-2000: decreasing mortality and the effect of provider caseload. Cancer 100:999-1007, 2004

2. Barrow DL: Subspecialization in neurosurgery. World Neurosurg 80:e105-e106, 2013

3. Bean JR: National healthcare spending in the U.S. and Japan: national economic policy and implications for neurosurgery. Neurol Med Chir (Tokyo) 45:18-24, 2005

4. Ekinci G, Akpinar IN, Baltacioğlu F, Erzen C, Kiliç T, Elmaci I, et al: Early-postoperative magnetic resonance imaging in glial tumors: prediction of tumor regrowth and recurrence. Eur J Radiol 45:99-107, 2003

5. Grey PL, Moffat DA, Palmer CR, Hardy DG, Baguley DM: Factors which influence the facial nerve outcome in vestibular schwannoma surgery. Clin Otolaryngol Allied Sci 21:409-413, 1996

6. Hansen D, Vedantam A, Briceño V, Lam SK, Luerssen TG, Jea A: Health-related quality of life outcomes and level of evidence in pediatric neurosurgery. J Neurosurg Pediatr 18:480-486, 2016

7. Herring B, Trish E: Explaining the growth in US health care spending using state-level variation in income, insurance, and provider market dynamics. Inquiry 52:4695801561897, 2015

8. Incekara F, Olubiyi O, Ozdemir A, Lee T, Rigolo L, Golby A: The value of pre- and intraoperative adjuncts on the extent of resection of hemispheric low-grade gliomas: a retrospective analysis. J Neurol Surg A Cent Eur Neurosurg 77:79-87, 2016

9. Isaacson B, Kileny PR, El-Kashlan H, Gadre AK: Intraoperative monitoring and facial nerve outcomes after vestibular schwannoma resection. Otol Neurotol 24:812-817, 2003

10. Kim SS, McCutcheon IE, Suki D, Weinberg JS, Sawaya R, Lang FF, et al: Awake craniotomy for brain tumors near eloquent cortex: correlation of intraoperative cortical mapping with neurological outcomes in 309 consecutive patients. Neurosurgery 64:13-16, 2009

11. Kunert P, Dziedzic T, Podgórska A, Czernicki T, Nowak A, Marchel A: Surgery for sporadic vestibular schwannoma. Part III: Facial and auditory nerve function. Neurol Neurochir Pol 49:373-380, 2015

12. Latif AZ, Signorini D, Gregor A, Whittle IR: The costs of managing patients with malignant glioma at a neuro-oncology clinic. Br J Neurosurg 12:118-122, 1998

13. Long DM, Gordon T, Bowman H, Etzel A, Burleyson G, Betchen S, et al: Outcome and cost of craniotomy performed to treat tumors in regional academic referral centers. Neurosurgery 52:1056-1065, 2003

14. Makary M, Chiocca EA, Erminy N, Antor M, Bergese SD, Abdel-Rasoul M, et al: Clinical and economic outcomes of low-field intraoperative MRI-guided tumor resection neurosurgery. J Magn Reson Imaging 34:1022-1030, 2011

15. Martino J, Gomez E, Bilbao JL, Dueñas JC, Vázquez-Barquero A: Cost-utility of maximal safe resection of WHO grade II gliomas within eloquent areas. Acta Neurochir (Wien) 155:41-50, 2013

16. Masera G, Baez F, Biondi A, Cavalli F, Conter V, Flores A, et al: North-South twinning in paediatric haemato-oncology: the La Mascota programme, Nicaragua. Lancet 352:19231926, 1998

17. Mirski MA, Chang CWJ, Cowan R: Impact of a neurosci- 
ence intensive care unit on neurosurgical patient outcomes and cost of care: evidence-based support for an intensivistdirected specialty ICU model of care. J Neurosurg Anesthesiol 13:83-92, 2001

18. O'Keeffe AB, Lawrence T, Bojanic S: Oxford Craniotomy Infections Database: a cost analysis of craniotomy infection. Br J Neurosurg 26:265-269, 2012

19. Ostrom QT, Gittleman H, Fulop J, Liu M, Blanda R, Kromer C, et al: CBTRUS Statistical Report: Primary Brain and Central Nervous System Tumors Diagnosed in the United States in 2008-2012. Neuro Oncol 17 (Suppl 4):iv1-iv62, 2015

20. Overcash M: A comparison of reusable and disposable perioperative textiles: sustainability state-of-the-art 2012. Anesth Analg 114:1055-1066, 2012

21. Pollom EL, Fujimoto DK, Han SS, Harris JP, Tharin SA, Soltys SG: Newly diagnosed glioblastoma: adverse socioeconomic factors correlate with delay in radiotherapy initiation and worse overall survival. J Radiat Res (Tokyo) 59 (Suppl 1):i11-i18, 2018

22. Ribeiro RC, Steliarova-Foucher E, Magrath I, Lemerle J, Eden T, Forget C, et al: Baseline status of paediatric oncology care in ten low-income or mid-income countries receiving My Child Matters support: a descriptive study. Lancet Oncol 9:721-729, 2008

23. Sanai N, Mirzadeh Z, Berger MS: Functional outcome after language mapping for glioma resection. N Engl J Med 358: $18-27,2008$

24. Seicean A, Seicean S, Schiltz NK, Alan N, Jones PK, Neuhauser D, et al: Short-term outcomes of craniotomy for malignant brain tumors in the elderly. Cancer 119:1058-1064, 2013

25. Shilpakar SK: Subspecialties in neurosurgery and its challenges in a developing country. World Neurosurg 75:335337, 2011

26. Slattery WH, Schwartz MS, Fisher LM, Oppenheimer M: Acoustic neuroma surgical cost and outcome by hospital volume in California. Otolaryngol Head Neck Surg 130:726735,2004

27. Sneh-Arbib O, Shiferstein A, Dagan N, Fein S, Telem L, Muchtar E, et al: Surgical site infections following craniotomy focusing on possible post-operative acquisition of infection: prospective cohort study. Eur J Clin Microbiol Infect Dis 32:1511-1516, 2013

28. Squires D, Anderson C: U.S. health care from a global perspective: spending, use of services, prices, and health in 13 countries. Issue Brief (Commonw Fund) 15:1-15, 2015
29. Sughrue ME, Yang I, Rutkowski MJ, Aranda D, Parsa AT: Preservation of facial nerve function after resection of vestibular schwannoma. Br J Neurosurg 24:666-671, 2010

30. Valduvieco I, Verger E, Bruna J, Caral L, Pujol T, Ribalta T, et al: Impact of radiotherapy delay on survival in glioblastoma. Clin Transl Oncol 15:278-282, 2013

31. Webster J, Alghamdi A: Use of plastic adhesive drapes during surgery for preventing surgical site infection. Cochrane Database Syst Rev (4):CD006353, 2015

32. Wilden JA, Voorhies J, Mosier KM, O'Neill DP, CohenGadol AA: Strategies to maximize resection of complex, or high surgical risk, low-grade gliomas. Neurosurg Focus 34(2):E5, 2013

33. World Bank: World Bank country and lending groups. World Bank. (https://datahelpdesk.worldbank.org/ knowledgebase/articles/906519-world-bank-country-andlending-groups) [Accessed August 8, 2018]

34. Yarascavitch BA, Chuback JE, Almenawer SA, Reddy K, Bhandari M: Levels of evidence in the neurosurgical literature: more tribulations than trials. Neurosurgery 71:11311138,2012

35. Zacharia BE, Deibert C, Gupta G, Hershman D, Neugut AI, Bruce JN, et al: Incidence, cost, and mortality associated with hospital-acquired conditions after resection of cranial neoplasms. Neurosurgery 74:638-647, 2014

\section{Disclosures}

The authors report no conflict of interest concerning the materials or methods used in this study or the findings specified in this paper.

\section{Author Contributions}

Conception and design: Abbassy. Acquisition of data: Helal, Abouzahra. Analysis and interpretation of data: Abbassy, Helal. Drafting the article: Abbassy, Helal. Critically revising the article: Abbassy, Helal, Fayed, Rayan. Reviewed submitted version of manuscript: Abbassy, Fayed, Rayan. Approved the final version of the manuscript on behalf of all authors: Abbassy. Study supervision: Abbassy.

\section{Correspondence}

Mahmoud Abbassy: Alexandria University, Alexandria, Egypt. mahmoud.abbassy@alexmed.edu.eg. 